\title{
BMJ Open Elucidating the association between regional variation in diagnostic frequency with risk-adjusted mortality through analysis of claims data of medicare inpatients: a cross- sectional study
}

\author{
Linyan Li, ${ }^{1,2}$ George F Chamoun, ${ }^{3}$ Nassib G Chamoun, ${ }^{3}$ Daniel Sessler, ${ }^{4}$ \\ Valérie Gopinath, ${ }^{5}$ Vikas Saini (i) ${ }^{5}$
}

To cite: Li L, Chamoun GF, Chamoun NG, et al. Elucidating the association between regional variation in diagnostic frequency with risk-adjusted mortality through analysis of claims data of medicare inpatients: a crosssectional study. BMJ Open 2021;11:e054632. doi:10.1136/ bmjopen-2021-054632

- Prepublication history and additional supplemental material for this paper are available online. To view these files, please visit the journal online (http://dx.doi.org/10.1136/ bmjopen-2021-054632)

Received 22 June 2021 Accepted 10 September 2021

Check for updates

(c) Author(s) (or their employer(s)) 2021. Re-use permitted under CC BY-NC. No commercial re-use. See rights and permissions. Published by BMJ.

For numbered affiliations see end of article.

Correspondence to

Vikas Saini;

vsaini@lowninstitute.org

\section{ABSTRACT}

Objective The validity of risk-adjustment methods based on administrative data has been questioned because hospital referral regions with higher diagnosis frequencies report lower case-fatality rates, implying that diagnoses do not track the underlying health risk. The objective of this study is to test the hypothesis that regional variation of diagnostic frequency in inpatient records is not associated with different coding practices but a reflection of the underlying health risks.

Design We applied two stratification methods to Medicare Analysis and Provider Review data from 2009 through 2014: (1) the number of chronic conditions; and, (2) quartiles of Risk Stratification Index (RSI)-defined risk. After sorting hospital referral regions into quintiles of diagnostic frequency, we examined all-cause mortality. Setting Medicare Analysis and Provider Review administrative database.

Participants 18126301 hospitalised Medicare fee-forservice beneficiaries aged 65 or older who had at least one hospital-based procedure between 2009 and 2014.

Exposure Coding frequency and baseline regional population risk factors by hospital referral region.

\section{Primary and secondary outcome(s) and} measure(s) One year all-cause mortality in patients having the same number of chronic conditions or within the same RSI score quartile across US health referral regions, grouped by diagnostic frequency.

Results No consistent relationship between diagnostic frequency and mortality in the risk stratum defined by number of chronic conditions was detected. In the strata defined by RSI quartile, there was no decrease in mortality as a function of diagnostic frequency. Instead, adjusted mortality was positively correlated with socioeconomic risk factors.

Conclusions Using present-on-admission codes only, diagnostic frequency among inpatients with at least one hospital-based procedure appears to be consequent to differences in baseline population health status, rather than diagnostic coding practices. In this population, claims-based risk-adjustment using RSI appears
Strengths and limitations of this study

- Our study uses inpatient, present-on-admission data to analyse diagnostic behaviour, which is robust and reliable.

- We compared different indicators of baseline health levels, including the number of chronic conditions, Risk Stratification Index and socioeconomic variables, rendering the conclusions well supported.

- The analysis was done for two separate years, which strengthens the validity of the results.

- Our analysis was restricted to the Medicare fee-forservice population.

- The census track data and Medicare data are from different years, potentially leading to some inaccurate estimates.

to be useful for assessing hospital outcomes and performance.

\section{INTRODUCTION}

Suitable risk-adjustment methods are necessary to fairly compare healthcare providers and systems ${ }^{1-7}$ Risk-adjustment systems based on administrative diagnostic codes are attractive because they are easy to use and the necessary data are readily available. However, significant regional variation exists for diagnostic code frequency. ${ }^{8-11}$ This puts the usage of diagnostic codes in question if the regional variation is due to differences in coding practices rather than true variation of underlying health status, and some investigators have expressed such concerns. ${ }^{8-12}$

In particular, Welch et al conducted a study to examine the relationship of regional variation of diagnostic code frequency to mortality. They analysed the relationship 
between case-fatality and regional diagnostic frequency in subgroups of population that have the same level of health risks, characterised by having the same number of nine major chronic conditions. The regional division used in the study was the hospital referral regions (HRRs), which were developed by the Dartmouth Atlas of Healthcare to depict the regional healthcare markets in the USA, defined based on where Medicare patients are most frequently referred for tertiary care. This study showed that the case-fatality was paradoxically lower in HRRs with higher diagnostic frequencies, for people with the same number of chronic conditions, implying that diagnostic codes carried less information about underlying health status in high-coding regions. ${ }^{11}$

While the results from that study suggested that diagnostic coding behaviour might undermine the reliability of claims-based risk-adjustment, there are some limitations that might weaken such a conclusion. First, Welch et al did not separate between inpatient and outpatient records. Inpatient coding is more standardised and uniform than outpatient coding, ${ }^{13}$ suggesting that inpatient populations might be less susceptible to regional coding variation. Second, using nine chronic conditions as an indicator of patients' underlying risk might be an over-simplification that might be susceptible to random variation; a more comprehensive and continuous riskassessment method might therefore yield different results.

In recent years, more advanced risk-adjustment methods have been developed that can more accurately quantify risk across large data sets. We tested the hypothesis that inpatient regional diagnostic frequency variation is not the consequence of diagnostic practice, but rather a reasonable reflection of regional baseline health. Specifically, we analysed geographic variation in the diagnostic frequency of chronic conditions and their relationship with mortality in a large national inpatient sample. We restrict our analysis to present-on-admission (POA) diagnostic codes, which are more robust and reliable. We adopted the method used by Welch et al using the number of chronic conditions as a crude measurement of health risk, ${ }^{14}$ and then compared it with a more comprehensive method-the Risk Stratification Index (RSI), a robust claims-based risk-assessment method. RSI was originally developed from a national Medicare sample of 35 million hospitalisations from 2001 to 2006, and was later validated and calibrated on 53101922 additional patients from the 2007-2012 Medicare data set. ${ }^{15}$ It builds logistic and Cox regression models to predict patients' health outcomes such as mortality and duration of hospitalisation, using input variables including patients' age, gender, procedural and diagnostic codes. The model achieved high accuracy and the output of the model can be used to reflect a patient's health risk. Finally, we assessed population variation in socioeconomic characteristics associated with health status and compared them to observed diagnostic frequencies.

\section{METHODS}

We used the 2009-2014 Medicare Analysis and Provider Review (MEDPAR) data set of Medicare beneficiaries, focusing on patients exceeding 65 years old who had at least one procedure. Only the initial admission across each year was considered for each patient. Diagnostic frequency was assessed within nine major chronic conditions based on the work of Iezzoni: ${ }^{14}$ cancer with poor prognosis, chronic obstructive pulmonary disease, coronary artery disease, congestive heart failure, peripheral artery disease, severe liver disease, diabetes with endorgan disease, chronic renal failure and dementia. The diagnostic codes corresponding to each chronic condition are provided in online supplemental table S1. Note that these codes have been processed following the procedure of Sessler $e t a t^{4}$ to include only representative ICD-9 codes that meet certain frequency criteria.

We tallied the number of chronic conditions for each initial admission during 2009-2014. We then computed the average diagnostic frequencies for 306 HRRs from 2009 and 2014. The average number of diagnosis codes within each stratum of chronic conditions in each year for all initial admission records was calculated for the entire study cohort, as well as within each HRR. Trends by year for each HRR were plotted to reflect annual variance and regional patterns. ${ }^{11}$

HRRs were divided into five groups based on the quintile values of the average diagnostic frequency of the entire study cohort. In order to assess the regional variation in diagnostic behaviour, we adopted the approach of Welch, ${ }^{11}$ but restricted to inpatient, POA records. We calculated age-adjusted and sex-adjusted all-cause mortalities in 2009 and 2014 in every quintile of HRRs. The mortalities are compared across quintiles of HRRs for the entire sample population, as well as for subgroups of population with $0,1,2$ and 3 chronic conditions, as a crude division of populations with similar levels of health risks.

We then repeated the analysis using the RSI method. Age-adjusted and sex-adjusted all-cause mortality was calculated for every quintile of HRR's for the entire sample population as well as four subgroups divided based on validated and calibrated RSI quartiles of risk for 1-year mortality, averaged for the population inside each subgroup.

In order to explore the potential reasons for diagnostic frequency variation in different HRRs, we analysed the regional patterns of some socioeconomic variables. These socioeconomic variables were extracted from a 2011/2012 census track database, including percentages of smoking, obesity, education more than college, African/BlackAmerican status, family household marriage status and median income. The smoking and obesity data were only available at the county level, while the data for other variables were obtained at zip code level. The average of each variable in each patient's home location was taken at the HRR level, and trend analysis is performed to elucidate the pattern of regional variation. 


\section{Statistical analysis}

We used various approaches to understand the relationship between diagnostic frequency and health outcomes. To test the association of mortality with diagnostic frequency for the entire population, we first sorted all HRRs in the USA into quintiles by diagnostic frequency (will abbreviate as 'HRR quintiles') and then computed the age-adjusted and sex-adjusted all-cause mortality for each HRR quintile group. This grouping method for HRRs is the same approach as used by Welch et al. ${ }^{9}$ To investigate whether the geographical variation in diagnostic frequency was due to different diagnostic labelling behaviours, we then also calculated age-adjusted and sexadjusted all-cause mortality for the patients at approximately the same health level (classified by number of chronic conditions or by RSI risk estimate) in each HRR quintile.

Health status was stratified into four groups by the number of chronic conditions (0-3) and by quartiles of RSI-defined risk. We calculated age-adjusted and sexadjusted all-cause mortality across the HRR quintiles within each stratum. Finally, we used various socioeconomic variables at the county level as population health indicators and examined their variation across HRR quintiles in comparison to the trend of variation for diagnostic frequency/RSI/mortality. Trends were tested by linear regressions using two-sided $\mathrm{p}$ values. $\mathrm{P}$ values less than 0.05 were considered statistically significant. The statistical analyses were performed using IBM SPSS Statistics V.22 and R V.3.2.3.

\section{Patient and public involvement statement}

Patient information was collected and stored in the US Centers for Medicare and Medicaid database. Patients and public were not directly involved in the design, conduct, reporting or dissemination of the research. Data are available from the US government.

\section{RESULTS}

The means and SD of the age and number of chronic conditions for the entire population (2009-2014) are $76.8 \pm 8.2$ and $1.31 \pm 1.18$, respectively. $44.9 \%$ of the study population are male and $55.1 \%$ are female. The values for specific years are shown in online supplemental table S2. The age distribution is relatively stable across the years. The diagnostic frequency is stable for years 2009 and 2010 , but then there was a $\approx 30 \%$ increase in 2011 with the number of reported chronic conditions thereafter remaining stable again. Regional variation in diagnostic frequencies across the USA was observed (figure 1).

\section{Primary outcome}

All-cause mortality for 2009 is shown in figure 2. For the entire population, the average diagnostic frequency was $1.09(\mathrm{SD}=0.989)$. There was a statistically significant but small increase in mortality from the lowest to the highest diagnostic frequency quintile: $0.149,0.155$, $0.160,0.157$ and $0.167(\mathrm{p}<0.001)$. In 2009, the proportion of people with $0,1,2$ and 3 chronic conditions was $32.1 \%, 37.3 \%, 21.4 \%$ and $7.5 \%$ respectively. The average mortality in each HRR quintile restricted to each of these groups of population are shown in figure 2 (upper figure). The mean $\pm \mathrm{SD}$ of mortality in these groups are $0.084 \pm 0.020$ (zero condition), $0.164 \pm 0.022$ (one condition), $0.211 \pm 0.024$ (two conditions) and $0.242 \pm 0.034$ (three conditions), respectively. It is unsurprising that mortality increases by large steps with increasing number of chronic conditions. For beneficiaries with 0 chronic conditions, there was a slight increasing trend of mortality with increasing coding frequency $(\mathrm{p}<0.001$, slope $=0.068$ mortality/diagnostic code. Unless otherwise mentioned, the unit for slopes in this paper is mortality per diagnostic code). For beneficiaries with 1, 2 or 3 chronic conditions, there was no monotonous trend for mortality ranging from the first to the fifth diagnostic frequency quintiles

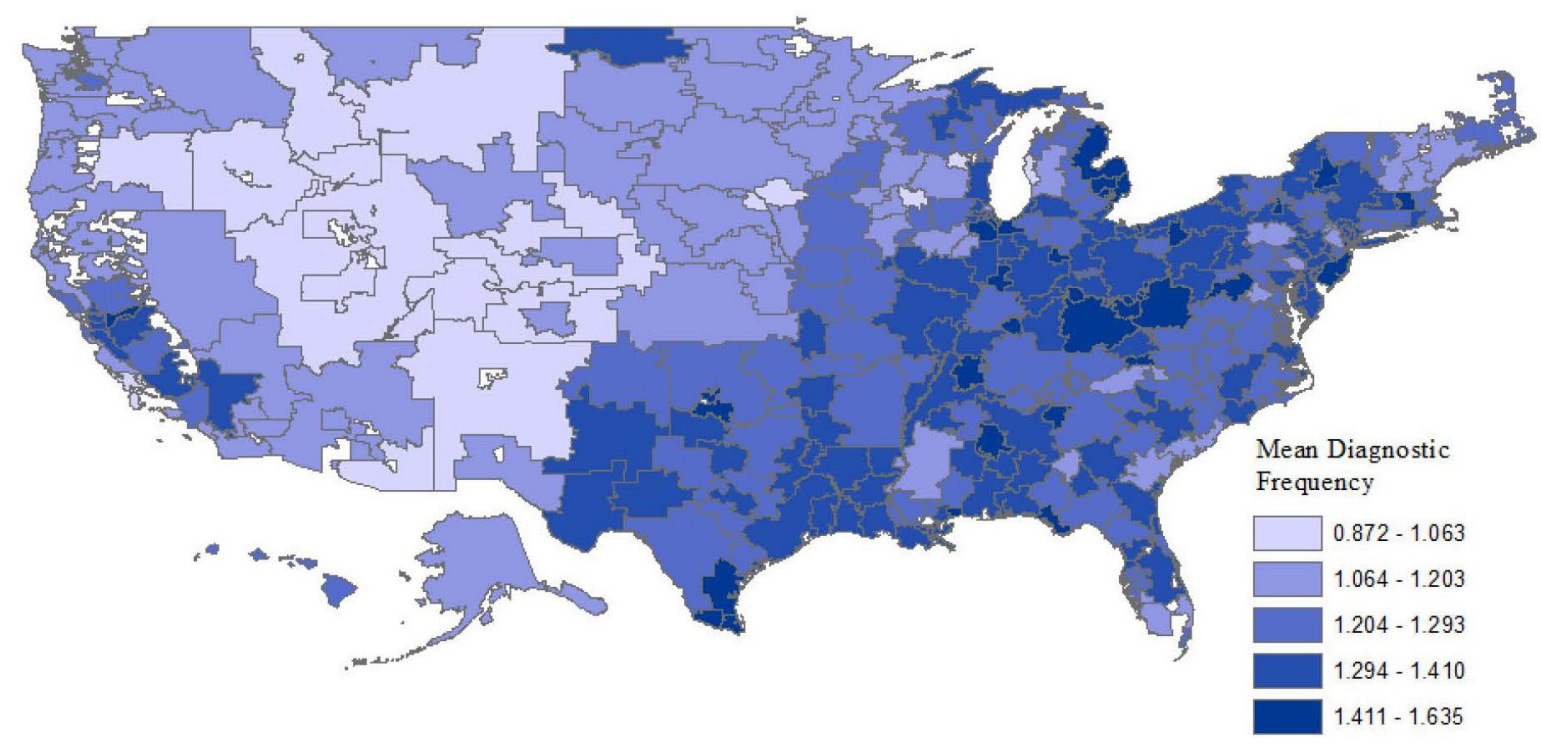

Figure 1 Regional map for mean diagnostic frequency (across years 2009-2014). 

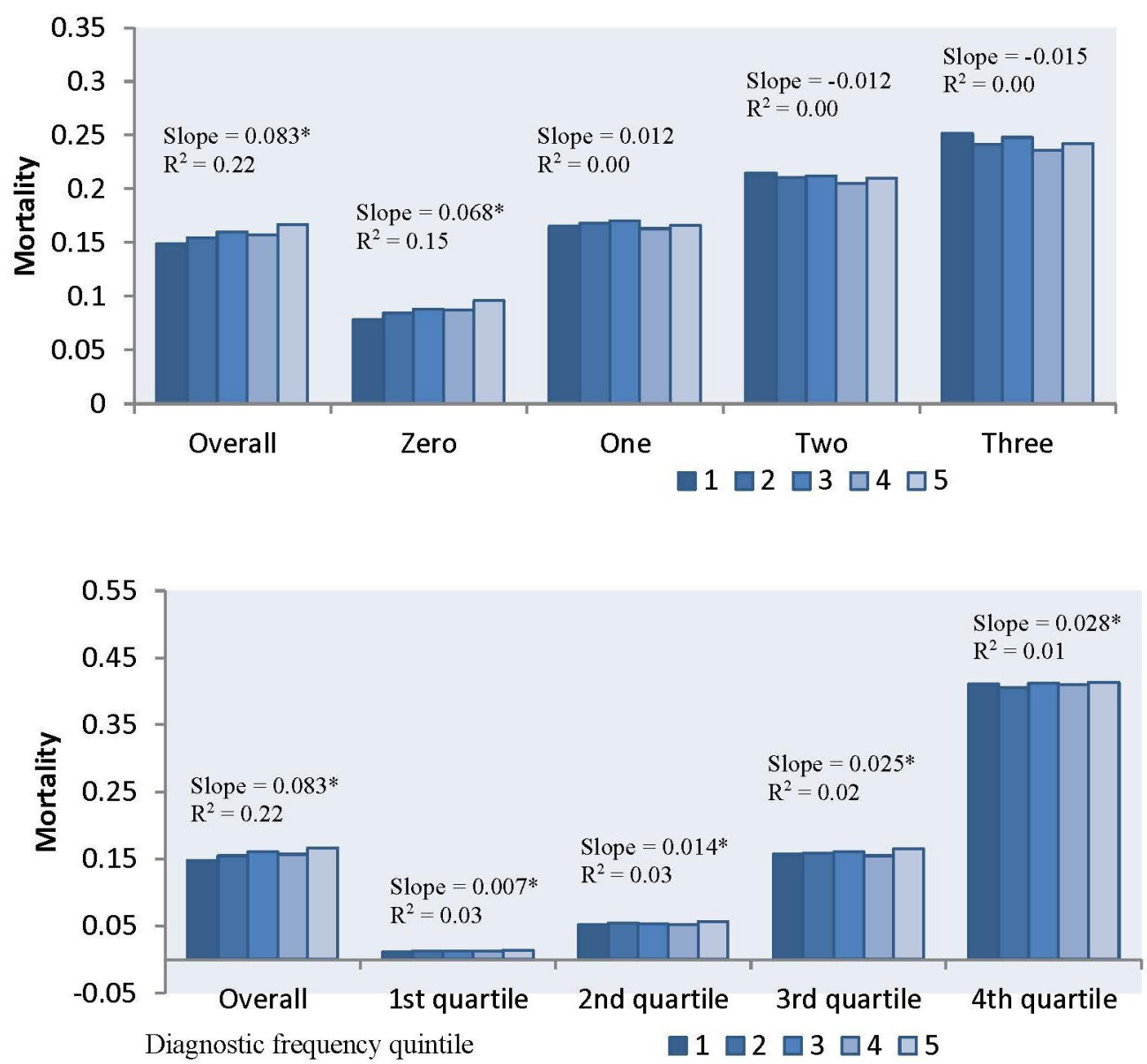

Figure 2 All-cause mortality in 2009 across the number of coded chronic conditions (upper figure) and quartiles of RSI risk (lower figure) stratified into quintiles of HRRs by diagnosis frequency (quintile 1: $\leq 1.01, n=578093$; quintile 2: 1.01-1.08, $n=572789$; quintile 3: 1.08-1.12, $n=619828$; quintile 4: $1.12-1.15, n=585870$; quintile $5:>1.15, n=596094)$. Slopes are obtained from linear regression of mortality and diagnostic frequency, with the unit of mortality/diagnostic code. ${ }^{*} \mathrm{P}<0.05$. HRRs, hospital referral regions; RSI, Risk Stratification Index.

by observation. The similar analysis stratifying the population into RSI risk quartiles instead of chronic conditions is shown in figure 2 (lower figure). The mean $\pm \mathrm{SD}$ of mortality in these groups are $0.013 \pm 0.004$ (first quartile), $0.054 \pm 0.010$ (second quartile), $0.161 \pm 0.018$ (third quartile) and $0.410 \pm 0.027$ (fourth quartile), respectively. Among the four RSI risk groups in 2009, there was a significant increasing trend within diagnostic frequency quintiles for all groups, but the slopes for the trends are much smaller than the slope for the entire population.

The results for 2014 are shown in figure 3. The average diagnostic frequency for the entire 2014 population was $1.42(\mathrm{SD}=1.26)$, with $27.8 \%, 30.8 \%, 21.8 \%$ and $12.6 \%$ of people having $0,1,2$ and 3 chronic conditions, respectively. The mean $\pm \mathrm{SD}$ of mortality in these groups are $0.054 \pm 0.013$ (zero condition), $0.146 \pm 0.019$ (one condition), $0.215 \pm 0.024$ (two conditions) and $0.270 \pm 0.031$ (three conditions), respectively. For the entire population, there was a significant trend for increasing mortality from the lowest diagnostic frequency quintile to the highest diagnostic frequency quintile $(0.147$, $0.164,0.166,0.164$ and 0.174 , respectively, $\mathrm{p}<0.001)$. For beneficiaries with no chronic conditions, there was an increasing trend of mortality with increasing coding frequency, but for beneficiaries with 1, 2 or 3 chronic conditions, there was no monotonous trend for mortality ranging from the first to the fifth diagnostic frequency quintiles by observation (figure 3). In 2014, similar trends to 2009 were observed, as mortality increased with HRR quintile higher in diagnostic frequency for all four RSI risk groups, but with slopes that were much lower than that of the overall population (figure 3 ). The mean \pm SD of mortality in these groups are $0.013 \pm 0.004$ (first quartile), $0.054 \pm 0.009$ (second quartile), $0.162 \pm 0.020$ (third quartile) and $0.436 \pm 0.030$ (fourth quartile), respectively.

The average levels of the socioeconomic variables for the population within each HRR are shown in figure 4, stratified by HRR quintiles. We found that obesity and smoking prevalence was greater in regions with higher diagnostic frequency quintile (slope $=0.089$ obesity rate / diagnostic code and 0.063 smoking rate/diagnostic code). Significant trends of increased percentage of black population (slope $=0.211$ black population percentage / diagnostic code) and population lacking higher education (slope $=0.112$ low education percentage $/$ diagnostic code), low median income $($ slope $=-\$ 9000 /$ diagnostic 


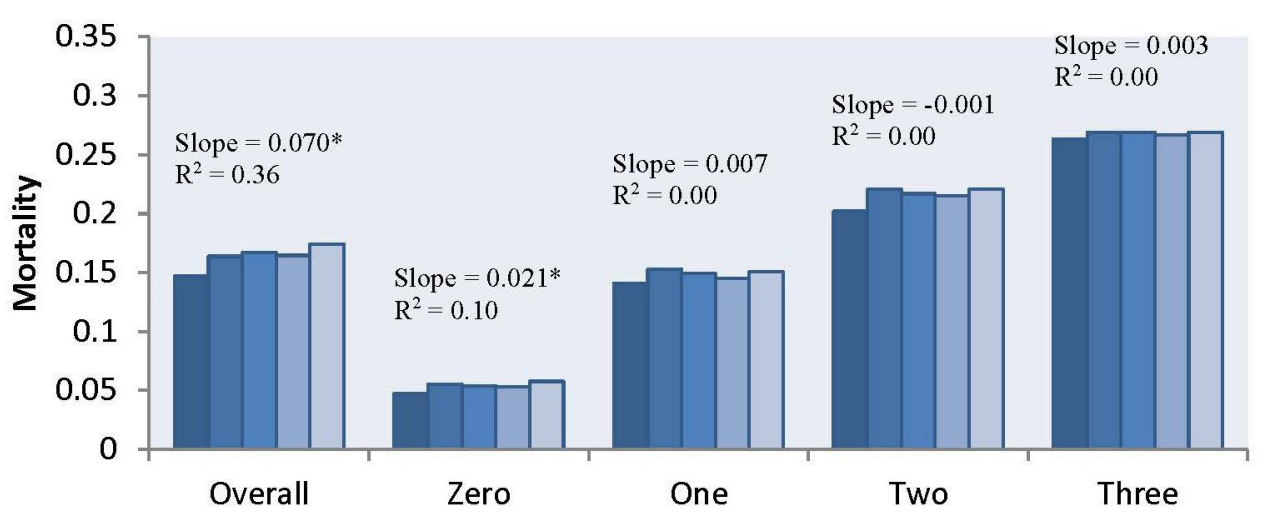

Diagnostic frequency quintile

$1 \square 2 \square 3 \square 4 \square 5$

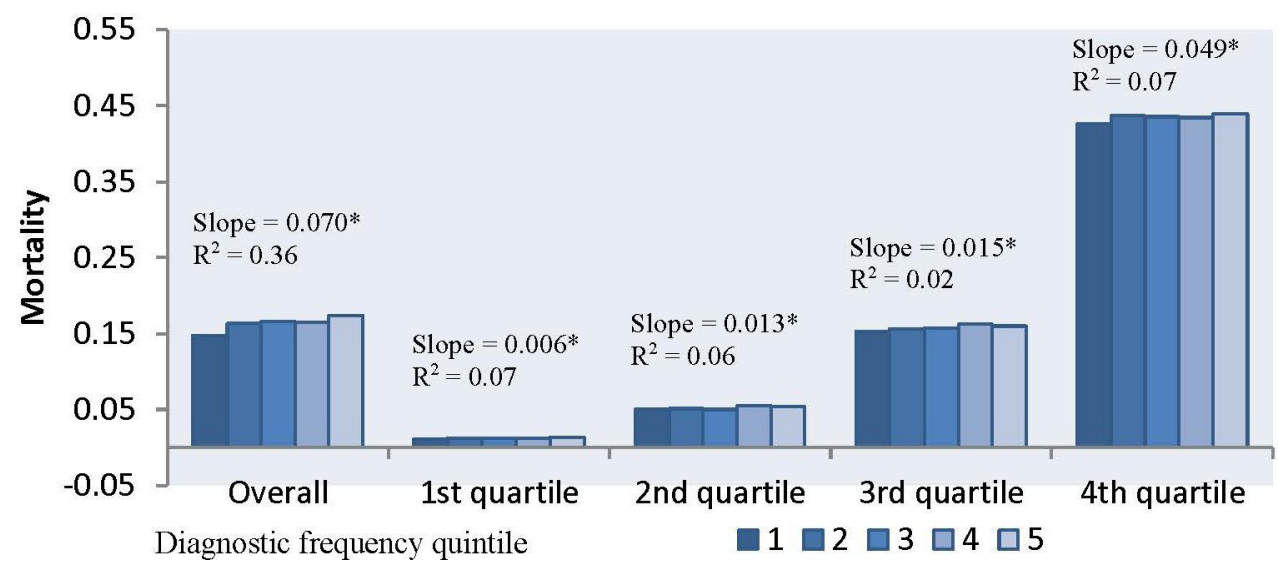

Figure 3 All-cause mortality in 2014 across the number of coded chronic conditions (upper figure) and quartiles of RSI risk (lower figure) stratified into quintiles of HRRs by diagnosis frequency (quintile 1: $\leq 1.32, n=558186$; quintile 2: 1.32-1.40, $n=640168$; quintile 3: $1.40-1.45, n=574205$; quintile 4: 1.45-1.51, $n=593417$; quintile $5:>1.51, n=604398$ ). Slopes are obtained from linear regression of mortality and diagnostic frequency, with the unit of mortality/diagnostic code. ${ }^{*} \mathrm{P}<0.05$. HRRs, hospital referral regions; RSI, Risk Stratification Index.

code) and low family households (slope=-0.069 family household percentage/diagnostic code) were also seen across quintiles of ascending diagnostic frequency. Baseline population health risk was thus likely to be worse in HRRs with higher diagnostic frequencies.

To further test the relationship between mortality and socioeconomic factors, we ran a multivariate regression between adjusted mortality in 2014 and the socioeconomic variables mentioned above. As shown in table 1, most of the socioeconomic variables are statistically significantly associated with mortality. We further added RSI as an additional covariate and the associations for most of the socioeconomic variables either become insignificant, or have a much smaller effect (the absolute value of the slope becomes much smaller). This indicates that RSI has adequately incorporated many aspects of socioeconomic status, and can be used as a valid risk-adjustment method.

\section{DISCUSSION}

We observed regional variation of diagnostic frequencies, as have many others. Some studies suggest that the variation can be explained by variation in patients' baseline health. Reschovsky, for example, concluded that $85 \%$ of the regional differences can be explained by variation in patient's baseline health after adjusting for possible bias in the use of claims-based health indicators. ${ }^{9}$ Others, though, argue that clinical diagnostic labelling behavioural patterns contribute most to regional variance. ${ }^{81112}$ For example, one study suggested that regional variation was not explained by disease prevalence in specialty outpatient settings. ${ }^{12}$ Another by Welch et al reported that case-fatality is lower in HRRs with higher diagnosis frequencies, implying the possibility of overcoding in high-coding regions. ${ }^{11}$ The difference in our study findings compared with Welch et al might be due to the different study population, and as such our findings properly apply only to the use of POA codes of inpatients that have at least one hospital procedure.

In our study, as might be expected, mortality increased as a function of the numbers of chronic conditions, and of higher RSI scores. Across diagnostic frequency quintiles, mortality for the whole population increased with 


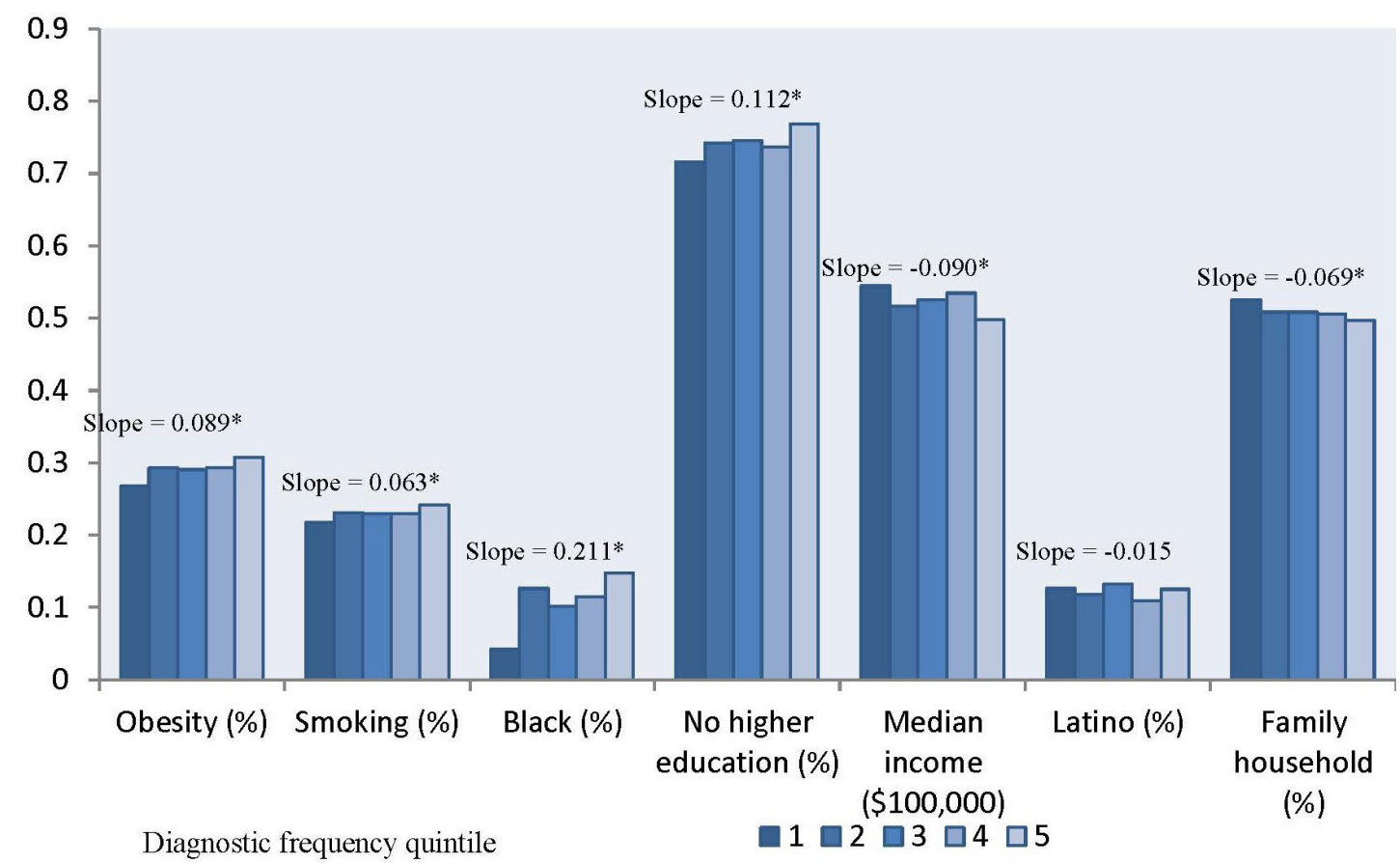

Figure 4 Social determinants (obesity, smoking, race, education†, median income, Latino and family household $\ddagger$ ) stratified into quintiles of HRRs by diagnosis frequency. ${ }^{*} \mathrm{P}<0.05$; †High education: percentage of people who have college education or above; łFamily household: percentage of households that are married family. HRRs, hospital referral regions.

increasing number of diagnoses. But for people with 1, 2 or 3 chronic conditions, we found either no statistically significant trend for mortality across quintiles of diagnostic frequency, or the trends were inconsistent for different years (eg, people with three chronic conditions show a negative trend in 2009, while a positive trend in 2014). In addition, the slopes of the trends were also generally small, with the largest magnitude being 0.049 mortality percentage per diagnostic code increase in coding frequency. Since the range of diagnostic frequency

Table 1 Linear regression of adjusted mortality in relation to socioeconomic variables

\begin{tabular}{|c|c|c|c|c|}
\hline & \multicolumn{2}{|c|}{ Model without RSI } & \multicolumn{2}{|c|}{ Model with RSI } \\
\hline & Slope & $P$ value & Slope & $P$ value \\
\hline $\mathrm{RSI}$ & - & - & $4.00 \mathrm{E}-01$ & $<0.001$ \\
\hline Obesity & 1.76E-04 & 0.747 & $-6.17 \mathrm{E}-04$ & 0.146 \\
\hline Smoking & $2.28 \mathrm{E}-03$ & $<0.001$ & $9.42 \mathrm{E}-04$ & 0.0143 \\
\hline Black $^{*}$ & 4.84E-02 & $<0.001$ & $2.30 \mathrm{E}-02$ & 0.0271 \\
\hline Not college† & 1.39E-01 & $<0.001$ & 4.57E-02 & 0.1047 \\
\hline Incomeł & $9.46 \mathrm{E}-07$ & $<0.001$ & $-1.57 \mathrm{E}-07$ & 0.368 \\
\hline Latino & 4.89E-02 & $<0.001$ & $8.56 \mathrm{E}-03$ & 0.2359 \\
\hline Not family§ & 2.24E-02 & 0.514 & $-1.40 \mathrm{E}-01$ & $<0.001$ \\
\hline
\end{tabular}

Two models are present, one without RSI as a covariate and one with RSI.

${ }^{*}$ The percentage of black people.

†The percentage of people with lower than college education. $\ddagger$ Median income.

§The percentage of population who are not in a family household. RSI, Risk Stratification Index. is around 0.2 , the trend represents less than $1 \%$ difference in mortality between the HRR with the highest and lowest diagnostic frequency, which is a relatively small variation. It is also worth noting that for people with zero chronic conditions, a consistent positive trend was observed both in 2009 and 2014. A possible interpretation is that for people with chronic conditions, their health risks were mainly dictated by these chronic conditions, and therefore people with the same number of chronic conditions showed similar mortality. For people without these chronic conditions, differences in other aspects of health could be reflected in mortality, so mortality was higher in regions with higher diagnostic frequency which indicates higher populational health risks. Based on this result, it would be worth examining the characteristics of the zero chronic condition group in more detail in additional studies, for example, whether they mainly comprise a very specific population (eg, inpatients admitted solely for elective procedures).

For people with similar health risks characterised by RSI, there is a slight increasing trend of mortality with regional diagnostic frequency. The slopes of the trends, again, are really small. Mortality was therefore similar for inpatients with comparable baseline health (defined by having the same number of chronic conditions or being in the same RSI quartile) irrespective of the average diagnostic frequency in their region. Regional diagnostic frequencies among inpatients therefore do not appear to be consequences of over-coding, but are instead more likely a consequence of higher baseline health risk. Our analysis of socioeconomic and behavioural risk factors further supports this since substantive differences in 
baseline health status are observed and can explain much of the observed geographical variations in mortality. These factors include known determinants of health outcomes such as smoking, low educational level, and poverty.

Comparing different measures of health risks, we further observed that mortality across HRR quintiles had smaller variations using RSI quartiles than when using the number of chronic conditions. We have found that inclusion of diagnostic codes in the RSI algorithm enhances the information content and risk-adjustment compared with methods with fewer and more broad categorisations of patient condition. ${ }^{16} 17$ Therefore, it is not surprising to find smaller variation in mortality using RSI quartiles compared with a simple count of chronic conditions.

The difference between RSI and other hierarchical conditions based models is that the risk variables admitted into RSI models are created as nested hierarchies, where children with low frequency of use are collapsed into close parents-effectively preserving the same condition but with less specificity. To be precise, 2495 codes are considered for each RSI regression model based on frequencies and truncation, and these codes encapsulate approximately $60 \%$ of the possible 21992 International Classification of Disease V.9 diagnostic and procedure codes. And among the $60 \%$ of the possible codes that were retained, the distribution of their weights is well balanced and smooth. ${ }^{4}$ In this way, the RSI 1-year mortality algorithm selects approximately 800 RSI risk variables that hierarchically encapsulate the information carried by 2348 codes. Thus, RSI has far greater resolution and stability than simply counting nine major disease codes, and it is therefore unsurprising that the method is more resistant to regional variations in coding frequency and performs better than tabulating the number of chronic conditions.

Our results contrast with those of Welch and colleagues whose work suggests that the prognostic significance of pooled inpatient and outpatient diagnostic codes varies in low-coding and high-coding regions. One likely explanation is that inpatient coding systems are more robust and uniform across regions than outpatient coding. We also note that coding behaviour as reflected in the MEDPAR data set appears to have become more uniform in 2014 compared with 2009, suggesting a possible convergence in regional coding practices over time even when confined to the inpatient population.

The $\approx 30 \%$ increase in diagnostic frequency after year 2011 is presumably consequent to an increase in the allowed number of codes from 9 to 25 that year. It is interesting to note that the increase in the diagnostic frequency at this time did not much affect regional diagnostic frequency rankings. However, since we observed some inconsistent trends for the same population across the years (eg, people with three chronic conditions), it would be desirable to further explore the effect of this coding policy change in future studies.

Our analysis was restricted to the Medicare fee-forservice population, with a minimum age of 65 and at least one procedural code. While we chose these criteria to define a more uniform study cohort when assessing the performance of a new algorithm (ie, the RSI), it is necessary to note that our results might not be generalisable to a different population, for example, the Medicare Advantage population, or the commercially insured population of younger patients. That said, RSI algorithms have been validated across various adult inpatient surgical populations and appear to be broadly applicable. ${ }^{48-20}$ Another limitation of our findings is that US census track data and Medicare data are not from identical populations, which may lead to inaccuracies in income estimates. Also, hospitals within a given region may have different coding practices which we would not have detected when considering entire referral regions. Finally, we restricted the analysis to POA codes to better reflect the short-term baseline risk of the patients. While there are POA exempt codes, these generally represent factors influencing health status that do not represent a current disease or injury or are always POA. As such, their contribution to short-term risk is likely smaller than the codes we included. We acknowledge that results might change if $\mathrm{POA}$ exempt codes were available, and this is a limitation of our analysis.

In summary, our study found that mortality is similar for inpatients with comparable baseline health risk, and no monotonous or consistent trends were observed for mortality with diagnostic frequency for these inpatients. Regional diagnostic frequencies among inpatients thus do not appear to be consequent to over-coding, but instead truly reflect higher baseline health risk. The RSI reliably tracked mortality, independently of regional coding practices and thus represents a promising method for adjusting baseline patient risk and comparing hospitals across various regions of the USA.

\section{Author affiliations}

${ }^{1}$ School of Data Science, City University of Hong Kong, Hong Kong, China

${ }^{2}$ Department of Environmental Health, Harvard T.H. Chan School of Public Health, Boston, Massachusetts, USA

${ }^{3}$ Health Data Analytics Institute, Dedham, Massachusetts, USA

${ }^{4}$ Outcomes Research, Cleveland Clinic, Cleveland, Ohio, USA

${ }^{5}$ Lown Institute, Needham, Massachusetts, USA

Twitter Vikas Saini @DrVikasSaini

Contributors NGC, DS and VS supervised the study design and obtained the data set; LL, GFC and VG performed data analysis; LL and VS prepared the manuscript.

Funding The authors have not declared a specific grant for this research from any funding agency in the public, commercial or not-for-profit sectors.

Map disclaimer The inclusion of any map (including the depiction of any boundaries therein), or of any geographic or locational reference, does not imply the expression of any opinion whatsoever on the part of BMJ concerning the legal status of any country, territory, jurisdiction or area or of its authorities. Any such expression remains solely that of the relevant source and is not endorsed by BMJ. Maps are provided without any warranty of any kind, either express or implied.

Competing interests LL, GFC, NGC and VG report no conflicts of interest. DS and VS have stock options in HDAl. DS also received consulting payments from HDAl and funding support to attend a work meeting at the Lown Institute.

Patient consent for publication Not required.

Ethics approval This study was granted a patient waiver of consent by the New England IRB under project reference W0 6484.

Provenance and peer review Not commissioned; externally peer reviewed. 
Data availability statement Data are available upon reasonable request.

Supplemental material This content has been supplied by the author(s). It has not been vetted by BMJ Publishing Group Limited (BMJ) and may not have been peer-reviewed. Any opinions or recommendations discussed are solely those of the author(s) and are not endorsed by BMJ. BMJ disclaims all liability and responsibility arising from any reliance placed on the content. Where the content includes any translated material, BMJ does not warrant the accuracy and reliability of the translations (including but not limited to local regulations, clinical guidelines, terminology, drug names and drug dosages), and is not responsible for any error and/or omissions arising from translation and adaptation or otherwise.

Open access This is an open access article distributed in accordance with the Creative Commons Attribution Non Commercial (CC BY-NC 4.0) license, which permits others to distribute, remix, adapt, build upon this work non-commercially, and license their derivative works on different terms, provided the original work is properly cited, appropriate credit is given, any changes made indicated, and the use is non-commercial. See: http://creativecommons.org/licenses/by-nc/4.0/.

ORCID iD

Vikas Saini http://orcid.org/0000-0001-9584-5548

\section{REFERENCES}

1 Ellis RP, Pope GC, lezzoni L, et al. Diagnosis-Based risk adjustment for Medicare capitation payments. Health Care Financ Rev 1996;17:101.

2 Quan $\mathrm{H}$, Li B, Couris CM, et al. Updating and validating the Charlson comorbidity index and score for risk adjustment in hospital discharge Abstracts using data from 6 countries. Am J Epidemiol 2011;173:676-82.

3 D'Hoore W, Sicotte C, Tilquin C. Risk adjustment in outcome assessment: the Charlson comorbidity index. Methods Inf Med 1993;32:382-7.

4 Sessler DI, Sigl JC, Manberg PJ, et al. Broadly applicable risk stratification system for predicting duration of hospitalization and mortality. Anesthesiology 2010;113:1026-37.

5 Pope GC, Kautter J, Ellis RP, et al. Risk adjustment of Medicare capitation payments using the CMS-HCC model. Health Care Financ Rev 2004;25:119.
6 Dalton JE, Glance LG, Mascha EJ, et al. Impact of presenton-admission indicators on risk-adjusted hospital mortality measurement. Anesthesiology 2013;118:1298-306.

7 Landon BE, Mechanic RE. The paradox of coding - policy concerns raised by risk-based provider contracts. N Engl J Med 2017;377:1211-3.

8 Song Y, Skinner J, Bynum J, et al. Regional variations in diagnostic practices. N Engl J Med 2010;363:45-53.

9 Reschovsky JD, Hadley J, Romano PS. Geographic variation in feefor-service medicare beneficiaries' medical costs is largely explained by disease burden. Med Care Res Rev 2013;70:542-63.

10 Keating NL, Landrum MB, Lamont EB, et al. Area-level variations in cancer care and outcomes. Med Care 2012;50:366-73.

11 Welch HG, Sharp SM, Gottlieb DJ, et al. Geographic variation in diagnosis frequency and risk of death among Medicare beneficiaries. JAMA 2011;305:1113-8.

12 Clough JD, Patel K, Shrank WH. Variation in specialty outpatient care patterns in the medicare population. J Gen Intern Med 2016;31:1278-86.

13 Yiannakoulias N, Svenson LW, Hill MD, et al. Regional comparisons of inpatient and outpatient patterns of cerebrovascular disease diagnosis in the province of Alberta. Chronic Dis Can 2003;24:9.

14 lezzoni LI, Heeren T, Foley SM, et al. Chronic conditions and risk of in-hospital death. Health Serv Res 1994;29:435.

15 Chamoun GF, Li L, Chamoun NG, et al. Validation and calibration of the risk stratification index. Anesthesiology 2017;126:623-30.

16 Chamoun GF, Li L, Chamoun NG, et al. Comparison of an updated risk stratification index to hierarchical condition categories. Anesthesiology 2018;128:109-16.

17 Sessler DI, Sigl JC, Manberg PJ, et al. Broadly applicable risk stratification system for predicting duration of hospitalization and mortality. Anesthesiology 2010;113:1026-37.

18 Gulack BC, Hale B, White WD, et al. Marriage and mortality after noncardiac surgery. J Surg Res 2017;210:152-8.

19 Sigakis MJG, Bittner EA, Wanderer JP. Validation of a risk stratification index and risk quantification index for predicting patient outcomes: in-hospital mortality, 30-day mortality, 1-year mortality, and length-of-stay. Anesthesiology 2013;119:525-40.

20 Wahl KM, Moretti E, White W, et al. Validation of a risk-stratification index for predicting 1-year mortality. Durham, Duke Univ Med Cent 2011. 